\title{
EDITORIAL
}

\section{The autonomous intelligence challenge}

\author{
Michel Cotsaftis
}

Undergraduate Scientific Research Program, ECE Paris, Paris, France

CORRESPONDING AUTHOR

Michel Cotsaftis, Undergraduate Scientific Research Program, ECE Paris, Paris, France; michel.cotsaftis@ece.fr

ARTICLE INFO

Received: May 31, 2017; Available online: August 3, 2017

The considerable development of modern technology during last decades has been accompanied by improvements in associated adapted machines' performance. The improvement process has always been guided by the same rules of researching higher efficiency and more secure effects each time. This leads to a progressive transfer of human action to more adapted and more specific effecting objects, from simple tools for elementary actions to more sophisticated machines for quite complex tasks. Each step of this transfer of human operator action has been realized by delegating to the effecting machine efficiency, accuracy, power and safety - basically all of technical nature and linked to power flux, with the human operator still keeping the mastery of the action to fulfil his own goals.

Today's phase, where performances expectation is largely overpassing human operator capacity, is now concerned with higher level of decision and coordination of action, i.e. of intelligence-based instead of on information flux, with consecutive machine autonomy, leaving a supervisory position to operator, until its (possible?) complete independence.

Finding the relations between power and information fluxes with the corresponding systems organization is today' fascinating and certainly longranging adventure at the forefront of theoretical and applied Engineering Science, to which the Journal of Autonomous Intelligence is willing to participate by opening its space to articles, reviews and lectures significantly contributing to the advancement in the understanding of these and related questions. To this aim, three main lines will be proposed by the Journal:
1) The understanding of higher decisional functions to be implemented in a machine.

2) The understanding of mechanisms leading from perception to intelligence and to decision.

3) The development of mathematical tools to represent and to carry the concepts developed in (1) and (2) up to their realization through valuable models.

The first is concerned with a bottom-up constructive approach, and the second is more adapted for top-down investigation, and the third is dealing with the setting of scientific framework within which the understanding is made efficient. Many research have been separately published along these three lines but often limited to a specific aspect, for instance, finding an "intelligent" controller (at mechanical level only), setting a retrieval process (at descriptive level only), or developing a communication scheme with disabled patients (at operating level only).

Here the main originality is the accent on global approach to the problem at concept level in order to provide the scientifically sound formalism for modeling and realizing the dumping of researched functionalities in the system. As easily seen, contributions in particular from engineering (control theory and robotic systems), applied mathematics (functional and numerical analysis, and complex system dynamics), neurosciences (brain signals analysis) and cell physiology (physicochemical dynamics) are equally welcomed for creating a community of research on these challenging topics all oriented toward intelligence transfer in view of system autonomy. 